\title{
Discrete unified gas kinetic scheme for multiscale anisotropic radiative heat transfer

\author{
Xinliang Song ${ }^{1}$, Chuang Zhang ${ }^{1}$, Xiafeng Zhou ${ }^{2}$ and Zhaoli Guo ${ }^{\text {* }}$
}

\author{
${ }^{*}$ Correspondence: \\ zlguo@hust.edu.cn \\ 'State Key Laboratory of Coal \\ Combustion, School of Energy and \\ Power Engineering, Huazhong \\ University of Science and \\ Technology, 430074 Wuhan, China \\ Full list of author information is \\ available at the end of the article
}

\begin{abstract}
In this work, a discrete unified gas kinetic scheme (DUGKS) is developed for radiative transfer in anisotropic scattering media. The method is an extension of a previous one for isotropic radiation problems [1]. The present scheme is a finite-volume discretization of the anisotropic gray radiation equation, where the anisotropic scattering phase function is approximated by the Legendre polynomial expansion. With the coupling of free transport and scattering processes in the reconstruction of the flux at cell interfaces, the present DUGKS has the nice unified preserving properties such that the cell size is not limited by the photon mean free path even in the optical thick regime. Several one- and two-dimensional numerical tests are conducted to validate the performance of the present DUGKS, and the numerical results demonstrate that the scheme is a reliable method for anisotropic radiative heat transfer problems.
\end{abstract}

Keywords: Gray radiative transfer equation, Anisotropic scattering, Scattering phase function, Legendre polynomial

\section{Introduction}

Radiative heat transfer appears in many engineering applications, such as short-pulsed laser in turbid media [2], radiative base heating from rocket exhaust plums [3], radiation in liquid rocket engines [4], nonequilibrium radiative hypersonic flows [5], and some other processes [6-9]. In practical applications, the scattering media are usually anisotropic [10], and some studies have been reported in the literature. For instance, it is found that radiative heat transfer can be greatly influenced in aerosol media due to the anisotropy properties [11]. The physically realistic approach for the scattering behaviour of coal combustion particles is the anisotropic, strongly forward scattering [12]. The liquid aluminum oxide particles produced from combustion of solid propellant exhibit a strong forward scattering characteristic [13].

Radiative heat transfer in anisotropic media can be described by the radiative transfer equation (RTE) for radiation intensity of the photon, which is a high dimensional integraldifferential equation. As the scattering effect of the media is strong (optical thick), i.e., the photon mean free path (MFP) $\lambda$ is much smaller than the characteristic macroscopic length $L$, the radiation behaves diffusively. On the other hand, the radiation can transport freely with the light speed when the scattering effect is weak (optical thin). Many practical media contain both strong and weak scattering regimes, such that it is necessary to design numerical schemes which can capture the radiation transport accurately in both

(c) The author(s). 2019 Open Access This article is distributed under the terms of the Creative Commons Attribution 4.0 International License (http://creativecommons.org/licenses/by/4.0/), which permits unrestricted use, distribution, and reproduction in any medium, provided you give appropriate credit to the original author(s) and the source, provide a link to the Creative Commons license, and indicate if changes were made. 
cases uniformly. The widely used stochastic Monte Carlo method (MCM) [14-16], which simulates the RTE by tracking the transport process of simulated photons with a mesh size smaller than the MFP, is inefficient in optical thick media. The classical deterministic discrete ordinates method (DOM) [17-19] and finite volume method (FVM) [10, 20 22] also suffer similar challenges. Therefore, it is necessary to develop multiscale schemes which are suitable for problems with arbitrary optical thickness without the limitation of the mesh size by the MFP.

The asymptotic preserving (AP) scheme for the linear kinetic equation is one of the idealized multiscale schemes. AP schemes are first studied in steady neutron transport problems [23-26]. For unsteady problems, some AP schemes have also been proposed $[27,28]$. Recently, an AP method, the unified gas kinetic scheme (UGKS) was successfully developed for radiative transfer problems [29-31]. Another asymptotic preserving multiscale method, the discrete unified gas kinetic scheme, which was initially designed for gas flows [32,33], was also extended to solve the radiative heat transfer problems in isotropic scattering media [1]. As the distribution function at a cell interface in DUGKS is constructed from the characteristic numerical solution rather than the local integral one in the UGKS, the DUGKS has a simpler structure and is more computational efficient. Furthermore, it can be shown that the DUGKS has the unified preserving properties such that it can serve as an efficient multiscale method [34]. However, both the UGKS and DUGKS have not considered the effects of the anisotropic scattering properties.

In present work, we will extend the DUGKS for isotropic radiative transfer to problems with anisotropic scattering effects. With the considering of the anisotropy, the radiative transfer features of the forward scattering media and the backward scattering media can be described clearly. The major difficulty of simulating the anisotropic problem stems from the change of the anisotropic scattering phase function in the RTE. In the present study, the anisotropic phase function will be calculated from the Legendre polynomial expansions [35, 36].

The remainder of this paper is organized as follows. Section 2 introduces the anisotropic scattering radiative transfer equation, and the DUGKS for RTE is described in Section 3. Some numerical tests are performed in Section 4. Finally, a brief summary is given in Section 5 .

\section{Gray radiative transfer equation}

The gray radiative transfer equation with anisotropic scattering reads [37, 38]

$$
\begin{aligned}
& \frac{1}{c} \frac{\partial I(\boldsymbol{x}, \boldsymbol{s}, t)}{\partial t}+\boldsymbol{s} \cdot \nabla I(\boldsymbol{x}, \boldsymbol{s}, t)=-\beta I(\boldsymbol{x}, \boldsymbol{s}, t)+\beta S(\boldsymbol{x}, \boldsymbol{s}, t), \\
& S(\boldsymbol{x}, \boldsymbol{s}, t)=(1-\omega) I_{b}(\boldsymbol{x}, t)+\frac{\omega}{4 \pi} \int_{4 \pi} I\left(\boldsymbol{x}, \boldsymbol{s}^{\prime}, t\right) \Phi\left(\boldsymbol{s}^{\prime}, \boldsymbol{s}\right) d \Omega^{\prime},
\end{aligned}
$$

where $I(\boldsymbol{x}, \boldsymbol{s}, t)$ is the distribution function of radiation intensity of photons, related to the spatial position $x$, the direction of photon propagation $s$ and time $t . c$ is the light speed, $\beta$ is the extinction coefficient which is the inverse of the local photon MFP, i.e., $\beta=1 / \lambda$. $S(\boldsymbol{x}, \boldsymbol{s}, t)$ is the source term of the RTE, and $\omega$ is the scattering albedo. The function $I_{b}(\boldsymbol{x}, t)$ is the blackbody intensity. For equilibrium radiative problems, the blackbody intensity can 
be calculated according to energy conservation,

$$
I_{b}(\boldsymbol{x}, t)=\frac{1}{4 \pi} \int_{4 \pi} I(\boldsymbol{x}, \boldsymbol{s}, t) d \Omega,
$$

where $\Omega$ is the solid angle domain of $s$. For radiative nonequilibrium problems, when the temperature field of the medium is given, the blackbody intensity can be calculated by the Stefan-Boltzmann law [37], i.e.,

$$
I_{b}(\boldsymbol{x}, t)=\frac{\sigma T^{4}(\boldsymbol{x}, t)}{\pi},
$$

where $\sigma$ is the Stefan-Boltzmann constant and $T$ is the local temperature of the medium, and $\Phi\left(s^{\prime}, s\right)$ is the scattering phase function, which describes the fraction of the radiative energy scattered into the outgoing direction $s$ from the incoming direction $\boldsymbol{s}^{\prime}$, and $\Omega^{\prime}$ is the corresponding solid angle domain. In the RTE, the anisotropic scattering characteristic of the participating media is fully expressed by the scattering phase function $\Phi\left(s^{\prime}, s\right)$, which satisfies the normalization condition,

$$
\frac{1}{4 \pi} \int_{4 \pi} \Phi\left(s^{\prime}, s\right) d \Omega^{\prime}=1 .
$$

Unlike the isotropic scattering problems where the scattering phase function is constant $(\Phi \equiv 1)$, the scattering phase function in anisotropic scattering problems changes according to the scattering angle. In this study it is approximated by a finite series of Legendre polynomials $[17,35,36]$, i.e.,

$$
\begin{aligned}
& \Phi\left(\boldsymbol{s}^{\prime}, \boldsymbol{s}\right)=\Phi(\cos \psi)=\sum_{j=0}^{N} C_{j}\left(\alpha_{1}, \alpha_{2}\right) P_{j}(\cos \psi), \\
& \cos \psi=\mu \mu^{\prime}+\left(1-\mu^{2}\right)^{1 / 2}\left(1-\mu^{\prime 2}\right)^{1 / 2} \cos \left(\varphi^{\prime}-\varphi\right),
\end{aligned}
$$

where $\psi$ is the angle between incoming direction $\boldsymbol{s}^{\prime}\left(\mu^{\prime}, \varphi^{\prime}\right)$ and scattered direction $s(\mu, \varphi), \mu$ is the cosine of the zenith angle and $\varphi$ is the azimuth angle of the direction $s$. $C_{j}\left(\alpha_{1}, \alpha_{2}\right)$ is the angular distribution coefficients, where $\alpha_{1}=\pi D / v$ and $\alpha_{2}=m \pi D / v$ with $D$ being the diameter of radiative medium particle, $v$ is the wavelength of the incident radiation in the surrounding medium, and $m$ is the complex index of refraction of radiative medium particle relative to the surrounding medium. $P_{j}$ is Legendre polynomials of order $j$. For strongly anisotropic scattering media, the upper limit $N$ should be big enough to ensure the accuracy of the calculation results.

In radiative transfer, the incident radiation energy $G$ and heat flux $q$ are two important physical quantities, which are defined from the radiation intensity,

$$
\begin{aligned}
& G=\int_{4 \pi} I(\boldsymbol{x}, \boldsymbol{s}, t) d \Omega, \\
& q=\int_{4 \pi} I(\boldsymbol{x}, \boldsymbol{s}, t) \boldsymbol{s} d \Omega .
\end{aligned}
$$

\section{Numerical scheme}

\subsection{DUGKS with anisotropic scattering effects}

In this section, the discrete unified gas kinetic scheme for gray radiative transfer equation involving the anisotropic scattering effects (Eq. (1)) is constructed in detail. Similar to the discretization approach in Ref [39], the solid angle space is discretized into $M$ discrete angles using the discrete ordinates method based on certain spherical quadratures, and 
correspondingly we obtain $M$ discrete directions $\boldsymbol{s}_{k}$. With these discrete directions, the RTE (1) can be expressed as

$$
\begin{aligned}
& \frac{1}{c} \frac{\partial I\left(\boldsymbol{x}, \boldsymbol{s}_{k}, t\right)}{\partial t}+\boldsymbol{s}_{k} \cdot \nabla I\left(\boldsymbol{x}, \boldsymbol{s}_{k}, t\right)=-\beta I\left(\boldsymbol{x}, \boldsymbol{s}_{k}, t\right)+\beta S\left(\boldsymbol{x}, \boldsymbol{s}_{k}, t\right), \\
& S\left(\boldsymbol{x}, \boldsymbol{s}_{k}, t\right)=(1-\omega) I_{b}(\boldsymbol{x}, t)+\frac{\omega}{4 \pi} \sum_{m=1}^{M}\left[I\left(\boldsymbol{x}, \boldsymbol{s}_{m}, t\right) \Phi\left(\boldsymbol{s}_{m}, \boldsymbol{s}_{k}\right) \omega_{m}\right],
\end{aligned}
$$

where $k, m=1,2, \ldots, M$, and $\omega_{m}$ is the weight assigned to the discretized direction $\boldsymbol{s}_{m}$. Following Refs. [1, 32], integrating Eq. (10) on a control volume $V_{j}$ centered at $\boldsymbol{x}_{j}$ from time $t_{n}$ to $t_{n+1}=t_{n}+\Delta t$, we can obtain

$$
\begin{aligned}
& I\left(\boldsymbol{x}_{j}, \boldsymbol{s}_{k}, t_{n+1}\right)-I\left(\boldsymbol{x}_{j}, \boldsymbol{s}_{k}, t_{n}\right)+\frac{c \Delta t}{\left|V_{j}\right|} F^{n+1 / 2} \\
& =\frac{c \beta \Delta t}{2}\left[S\left(\boldsymbol{x}_{j}, \boldsymbol{s}_{k}, t_{n+1}\right)-I\left(\boldsymbol{x}_{j}, \boldsymbol{s}_{k}, t_{n+1}\right)\right]+\frac{c \beta \Delta t}{2}\left[S\left(\boldsymbol{x}_{j}, \boldsymbol{s}_{k}, t_{n}\right)-I\left(\boldsymbol{x}_{j}, \boldsymbol{s}_{k}, t_{n}\right)\right],
\end{aligned}
$$

where

$$
F^{n+1 / 2}=\sum_{f}\left(\boldsymbol{s}_{k} \cdot \boldsymbol{n}_{f}\right) I\left(\boldsymbol{x}_{f}, \boldsymbol{s}_{k}, t_{n+1 / 2}\right) \triangle S_{f},
$$

is the flux across the cell interface, $I\left(\boldsymbol{x}_{j}, \boldsymbol{s}_{k}, t_{n}\right)$ denotes the cell averaged value for the diffuse intensity at time $t_{n}$ with control volume of $V_{j}$ located at $x_{j}$ along photon propagation direction $\boldsymbol{s}_{k}, \boldsymbol{n}_{f}$ is the outward unit normal vector at $\boldsymbol{x}_{f}$ of an interface, and $\Delta S_{f}$ is the corresponding interface area. The midpoint rule for the integration of the second term on the left-hand of Eq. (12) and trapezoidal rule for the right-hand of Eq. (12) are used, respectively. Two new distribution functions are introduced to remove the implicitness in Eq. (12),

$$
\begin{aligned}
& \widetilde{I}(\boldsymbol{x}, \boldsymbol{s}, t)=I(\boldsymbol{x}, \boldsymbol{s}, t)+\frac{\chi}{2}[I(\boldsymbol{x}, \boldsymbol{s}, t)-S(\boldsymbol{x}, \boldsymbol{s}, t)], \\
& \widetilde{I}^{+}(\boldsymbol{x}, \boldsymbol{s}, t)=I(\boldsymbol{x}, \boldsymbol{s}, t)-\frac{\chi}{2}[I(\boldsymbol{x}, \boldsymbol{s}, t)-S(\boldsymbol{x}, \boldsymbol{s}, t)],
\end{aligned}
$$

where $\chi=c \beta \triangle t$. Then Eq. (12) can be rewritten as

$$
\widetilde{I}\left(\boldsymbol{x}_{j}, \boldsymbol{s}_{k}, t_{n+1}\right)=\widetilde{I}^{+}\left(\boldsymbol{x}_{j}, \boldsymbol{s}_{k}, t_{n}\right)-\frac{c \Delta t}{\left|V_{j}\right|} F^{n+1 / 2} .
$$

In order to evaluate the cell interface flux at the half time-step $F^{n+1 / 2}$, we integrate Eq. (10) along the characteristic line with a half time step,

$$
\begin{aligned}
& I\left(\boldsymbol{x}_{f}, \boldsymbol{s}_{k}, t_{n+1 / 2}\right)-I\left(\boldsymbol{x}_{f}-\boldsymbol{s}_{k} c h, \boldsymbol{s}_{k}, t_{n}\right) \\
& =\frac{c \beta h}{2}\left[S\left(\boldsymbol{x}_{f}, \boldsymbol{s}_{k}, t_{n+1 / 2}\right)-I\left(\boldsymbol{x}_{f}, \boldsymbol{s}_{k}, t_{n+1 / 2}\right)\right] \\
& +\frac{c \beta h}{2}\left[S\left(\boldsymbol{x}_{f}-\boldsymbol{s}_{k} c h, \boldsymbol{s}_{k}, t_{n}\right)-I\left(\boldsymbol{x}_{f}-\boldsymbol{s}_{k} c h, \boldsymbol{s}_{k}, t_{n}\right)\right],
\end{aligned}
$$

where $h=\Delta t / 2$, and the trapezoidal rule is again used to evaluate the right-hand term of Eq. (10). Another two new distribution functions are also introduced to remove the implicitness in Eq. (17),

$$
\begin{aligned}
& \bar{I}(\boldsymbol{x}, \boldsymbol{s}, t)=I(\boldsymbol{x}, \boldsymbol{s}, t)+\frac{\chi}{4}[I(\boldsymbol{x}, \boldsymbol{s}, t)-S(\boldsymbol{x}, \boldsymbol{s}, t)], \\
& \bar{I}^{+}(\boldsymbol{x}, \boldsymbol{s}, t)=I(\boldsymbol{x}, \boldsymbol{s}, t)-\frac{\chi}{4}[I(\boldsymbol{x}, \boldsymbol{s}, t)-S(\boldsymbol{x}, \boldsymbol{s}, t)] .
\end{aligned}
$$


Substituting Eqs. (18) and (19) into Eq. (17), we can obtain

$$
\bar{I}\left(\boldsymbol{x}_{f}, \boldsymbol{s}_{k}, t_{n+1 / 2}\right)=\bar{I}^{+}\left(\boldsymbol{x}_{f}-\boldsymbol{s}_{k} c h, \boldsymbol{s}_{k}, t_{n}\right) .
$$

$\bar{I}^{+}\left(\boldsymbol{x}_{f}-\boldsymbol{s}_{k} c h, \boldsymbol{s}_{k}, t_{n}\right)$ can be reconstructed by

$$
\begin{array}{r}
\bar{I}^{+}\left(\boldsymbol{x}_{f}-\boldsymbol{s}_{k} c h, \boldsymbol{s}_{k}, t_{n}\right)=\bar{I}^{+}\left(\boldsymbol{x}_{j}, \boldsymbol{s}_{k}, t_{n}\right)+\left(\boldsymbol{x}_{f}-\boldsymbol{s}_{k} c h-\boldsymbol{x}_{j}\right) \cdot \sigma_{j}, \\
\left(\boldsymbol{x}_{f}-\boldsymbol{s}_{k} c h\right) \in V_{j},
\end{array}
$$

where $\sigma_{j}$ is the slope of the distribution function $\bar{I}^{+}$in cell $j$. In the present study, the van Leer limiter [40] is used to calculate the slope.

The new distribution functions $\widetilde{I}, \widetilde{I}^{+}, \bar{I}, \bar{I}^{+}$are all related to the original distribution function $I$ and the scattering phase function $\Phi(\cos \psi)$. Their relations in the present work can be finally obtained as

$$
\begin{aligned}
I(\boldsymbol{x}, \boldsymbol{s}, t) & =\frac{4}{4+\chi} \bar{I}(\boldsymbol{x}, \boldsymbol{s}, t)+\frac{\chi}{4+\chi}(1-\omega) I_{b}(\boldsymbol{x}, t) \\
& +\frac{\chi \omega}{4 \pi(4+\chi)} \int_{4 \pi} I\left(\boldsymbol{x}, \boldsymbol{s}^{\prime}, t\right) \Phi\left(\boldsymbol{s}^{\prime}, \boldsymbol{s}\right) d \Omega^{\prime}, \\
\bar{I}^{+}(\boldsymbol{x}, \boldsymbol{s}, t) & =\frac{4-\chi}{4+2 \chi} \widetilde{I}(\boldsymbol{x}, \boldsymbol{s}, t)+\frac{3 \chi}{4+2 \chi}(1-\omega) I_{b}(\boldsymbol{x}, t) \\
& +\frac{\omega \chi}{8 \pi(2+\chi)} \int_{4 \pi}\left[\widetilde{I}(\boldsymbol{x}, \boldsymbol{s}, t)+2 \bar{I}^{+}(\boldsymbol{x}, \boldsymbol{s}, t)\right] \Phi\left(\boldsymbol{s}^{\prime}, \boldsymbol{s}\right) d \Omega^{\prime}, \\
\widetilde{I}^{+}(\boldsymbol{x}, \boldsymbol{s}, t) & =\frac{4}{3} \bar{I}^{+}(\boldsymbol{x}, \boldsymbol{s}, t)-\frac{1}{3} \widetilde{I}(\boldsymbol{x}, \boldsymbol{s}, t) .
\end{aligned}
$$

In the isotropic scattering condition $(\Phi \equiv 1)$, Eqs. (22) and (23) can be solved explicitly [1]. However, in anisotropic case, due to the complexity of the phase function $\left(\Phi\left(\boldsymbol{s}^{\prime}, \boldsymbol{s}\right)\right)$, Eqs. (22) and (23) can not be solved explicitly. Here a simple iterative method is employed as follows. First, the iteration procedure for the calculation of the original distribution function $I\left(\boldsymbol{x}_{f}, \boldsymbol{s}_{k}, t_{n+1 / 2}\right)$ is

$$
\begin{aligned}
I^{l+1}\left(\boldsymbol{x}_{f}, \boldsymbol{s}_{k}, t_{n+1 / 2}\right) & =\frac{4}{4+\chi} \bar{I}\left(\boldsymbol{x}_{f}, \boldsymbol{s}_{k}, t_{n+1 / 2}\right)+\frac{\chi}{4+\chi}(1-\omega) I_{b}{ }^{l}\left(\boldsymbol{x}_{f}, t_{n+1 / 2}\right) \\
& +\frac{\chi \omega}{4 \pi(4+\chi)} \sum_{m=1}^{M} \sum_{j=0}^{N} \omega_{m}\left[I^{l}\left(\boldsymbol{x}_{f}, \boldsymbol{s}_{m}, t_{n+1 / 2}\right) C_{j} P_{j}(\cos \psi)\right],
\end{aligned}
$$

where $l$ is the iteration index, and

$$
I_{b}{ }^{l}\left(\boldsymbol{x}_{f}, t_{n+1 / 2}\right)=\frac{1}{4 \pi} \sum_{k=1}^{M} \omega_{k} I^{l}\left(\boldsymbol{x}_{f}, \boldsymbol{s}_{k}, t_{n+1 / 2}\right) .
$$

The iteration stops as the intensity is converged, i.e., $\left|I^{l+1}-I^{l}\right|<\epsilon$, where $\epsilon$ is a small number which is set to be $10^{-4}$ in our simulations. Eq. (23) is also solved iteratively in a similar way. We found that the number of iterations is less than 10 in almost all the numerical tests in the present work. But for more complex problems, more advanced acceleration technique should be employed.

Finally, we note that with the use of the trapezoidal rule in Eqs. (12) and (17), the present DUGKS is a semi-implicit scheme and the time step $\Delta t$ is not limited by the scattering, which is determined by the Courant-Friedrichs-Lewy (CFL) condition [41],

$$
\Delta t=\alpha \frac{\Delta x}{c},
$$

where $0<\alpha<1$ is the CFL number, and $\Delta x$ is the minimal grid spacing. 


\subsection{Boundary conditions}

In the present work, diffusely emitting and reflecting boundaries are considered. When the wall is black, a photon is absorbed as it hits the wall, and a new photon in thermal equilibrium with boundary temperature is emitted into the domain. When the wall is gray, some of the incident photons are absorbed and the rest are reflected diffusively back to the domain, depending on the reflectivity of the wall. The general boundary condition for Eq. (1) can be expressed as

$$
I\left(\boldsymbol{x}_{w}, \boldsymbol{s}, t\right)=\varepsilon_{w} I_{b}\left(\boldsymbol{x}_{w}\right)+\frac{\rho_{w}}{\pi} \int_{\boldsymbol{n}_{w} \cdot \boldsymbol{s}^{\prime}<0}\left(\boldsymbol{n}_{w} \cdot \boldsymbol{s}^{\prime}\right) I\left(\boldsymbol{x}_{w}, \boldsymbol{s}^{\prime}, t\right) d \Omega^{\prime},
$$

where $\varepsilon_{w}$ is the diffuse emissivity, $\rho_{w}$ is the diffuse reflectivity, and $\boldsymbol{n}_{w}$ is the unit inner normal vector at the boundary. $I_{b}\left(\boldsymbol{x}_{w}\right)$ is the blackbody radiation intensity at the boundary surface having a specified temperature. This boundary condition is implemented in the DUGKS straightforwardly by replacing the $s$ with each discrete angle $s_{k}$, and evaluating the integral with the numerical quadrature.

\subsection{Algorithm}

In summary, the main procedure of the DUGKS from time step $t_{n}$ to $t_{n+1}$ can be summarized as follows:

1. Calculate the microflux $\boldsymbol{F}^{n+1 / 2}$ at cell interface $\boldsymbol{x}_{f}$ and at time $t_{n+1 / 2}$.

(a) Calculate $\bar{I}^{+}$from $\widetilde{I}$ at each cell center with the iterative method according to Eq. (23);

(b) Reconstruct the slope $\sigma_{j}$ of $\bar{I}^{+}$in each cell center;

(c) Reconstruct the distribution function $\bar{I}^{+}$at $x_{f}-\boldsymbol{s}_{k} c h$ according to Eq. (21);

(d) Calculate the distribution function $\bar{I}$ at cell interface at time $t_{n+1 / 2}$ according to Eq. (20);

(e) Calculate the original distribution function $I$ at cell interface and at time $t_{n+1 / 2}$ with the iterative method according to Eq. (25);

(f) Calculate the microflux $\boldsymbol{F}^{n+1 / 2}$ through each cell interface from $I\left(\boldsymbol{x}_{f}, \boldsymbol{s}_{k}, t_{n+1 / 2}\right)$ according to Eq. (13).

2. Calculate $\widetilde{I}^{+}$at cell center and at time $t_{n}$ according to Eq. (24).

3. Update the cell averaged $\widetilde{I}$ in each cell from $t_{n}$ to $t_{n+1}$ according to Eq. (16).

When the transformed intensity distribution is known, the local incident radiation energy can be calculated based on Eq. (14)

$$
G(x, t)=\frac{2}{2+\chi(1-\omega)} \sum_{k=1}^{M} \omega_{k} \tilde{I}\left(\boldsymbol{x}, s_{k}, t\right)+\frac{\chi(1-\omega)}{2+\chi(1-\omega)} 4 \pi I_{b}(x, t),
$$

and the net radiative heat flux can be calculated based on Eqs. (14) and (19),

$$
\left.q(\boldsymbol{x}, t)=\frac{1}{3} \sum_{k=1}^{M}\left[\omega_{k} \boldsymbol{s}_{k} \widetilde{I}\left(\boldsymbol{x}, \boldsymbol{s}_{k}, t\right)+2 \bar{I}^{+}\left(\boldsymbol{x}, \boldsymbol{s}_{k}, t\right)\right)\right] .
$$

\section{Numerical examples}

In this section, three radiative transfer problems in anisotropic scattering media are simulated to validate the proposed DUGKS, including the radiative transfer in a slab with different wall temperatures, radiative transfer in a square domain with a hot wall, and 
radiative transfer in a square domain with collimated incidence. In each case, Cartesian coordinates is used to discretize the physical space, and the Gauss-Legendre quadrature [42] is used for angular discretization, where $\mu \in[-1,1]$ and $\varphi \in[0,2 \pi]$ are the cosine of zenith and azimuth angle, respectively. The CFL number is taken to be $\alpha=0.5$. For steady problems, the system is regarded as converged as $E<10^{-6}$, where

$$
E=\frac{\sum_{i, j}\left|G_{i, j}^{n}-G_{i, j}^{n+1000}\right|}{\sum_{i, j}\left|G_{i, j}^{n}\right|} .
$$

\subsection{Radiation in a slab with different wall temperatures}

In the first case, the DUGKS is applied to the radiative heat transfer in a slab with thickness $L$ filled with anisotropic absorbing-scattering media, as shown in Fig. 1 . The temperatures on the boundaries located at $x=0$ and $x=L$ are maintained at $T_{0}$ and $T_{1}$, respectively, where $T_{1}>T_{0}$. The angular space is discretized into 40 control angles. The physical space is discretized into $N_{x}=21$ uniform cells. Two anisotropic media composed of different particle clouds are considered, where the radiative properties are summarized in Table 1 . The scattering phase function is expressed by $N$-th Legendre polynomial expansions obtained from Eq. (6). Figure 2 shows the non-dimensional radiation energy $\Phi_{b}$ at different optical thickness $\tau=\beta L$, where $\Phi_{b}=\left(G-G_{0}\right) /\left(G_{1}-G_{0}\right)$ with $G_{0}=4 \sigma T_{0}^{4}$ and $G_{1}=4 \sigma T_{1}^{4}$. It can be seen that the results are in good agreement with the exact results for $\tau=0.1,1,5$ [43]. When $\tau$ is large, the incident radiation energy $G$ can be solved by the limit diffusion equation such that the distribution of $G$ is linear, where the optical thick cases for $\tau=21,40,100,1000$ agree well with that. It is noted that the results are still satisfied even as $\Delta x / \lambda=\tau / N_{x} \geq 1$. These results confirm the capability of the present DUGKS method in simulating anisotropic heat transfer process for one-dimensional problems.

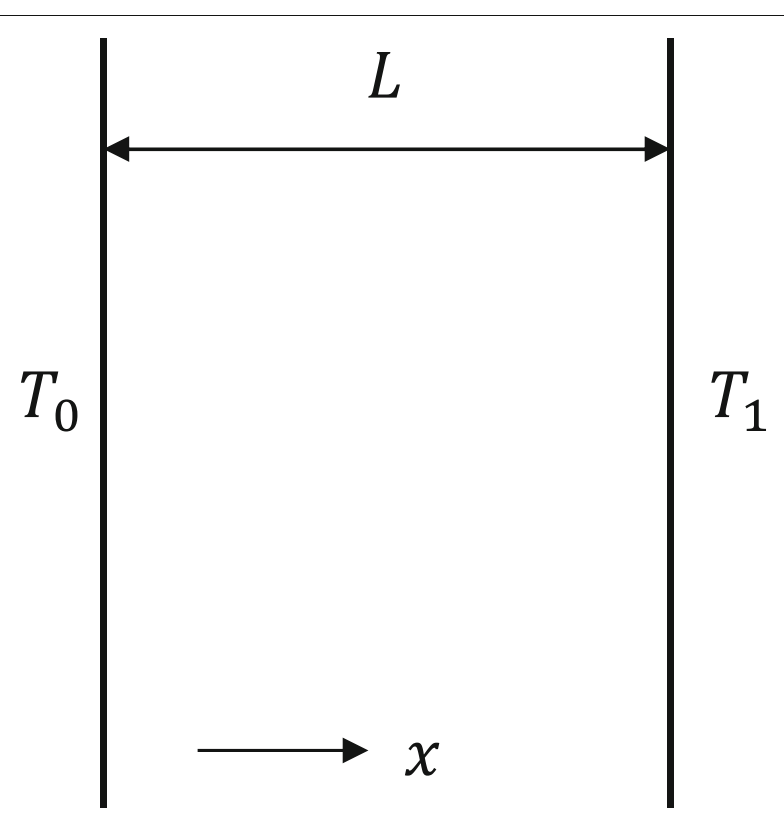

Fig. 1 Schematic of one-dimensional radiation heat transfer 
Table 1 Radiative properties of anisotropic absorbing scattering particle clouds

\begin{tabular}{llllll}
\hline media & $D$ & $m$ & $\beta$ & $\omega$ & orders N \\
\hline A & 5 & $2-i$ & 0.01904 & 0.5634 & 26 \\
B & 5 & 2 & 0.06420 & 1 & 27 \\
\hline
\end{tabular}

\subsection{Radiation in a square domain with a hot wall}

In this subsection, we consider a square with the side length of $L$ enclosed by four boundaries, as shown in Fig. 3. The bottom wall is kept hot with a non-dimensional temperature of $T_{1}=1$, while the other walls and the media are kept cold with a non-dimensional temperature of $T_{0}=0$.

A grid size of $N_{x} \times N_{y}=26 \times 26$ is used for physical space discretization. The direction cosine of zenith angle $\mu \in[-1,1]$ is discretized with $N_{\mu}=16$ points, while the azimuth angle $\varphi \in[0,2 \pi]$ is discretized into $N_{\varphi}=16$ points. The scattering albedo is set to be $\omega=1.0$. Four different kinds of anisotropic scattering media and the isotropic scattering medium are considered. Table 2 shows the expansion coefficients $C_{j}$ and the asymmetry factors $C_{j} / 3$ of the phase function for different scattering media [17]. The anisotropic and isotropic scattering phase functions varying with the scattering angle are shown in Fig. 4. It can be observed that the phase function for the anisotropic scattering media changes dramatically with the change of the scattering angle, which will significantly influence the energy transfer in practical problems.

Figure 5 shows the net radiative heat flux in the $y$-direction along the vertical centerline at $x=L / 2$. The net hot surface radiative heat flux is shown in Fig. 6 . For comparison, we also present the solutions of DOM [17] for this problem. It can be seen the DUGKS results agree quite well with the DOM results for five different media. From Fig. 5 and Fig. 6, the effects of the anisotropic scattering media can be seen clearly. The forward scattering media transport more radiation heat into the forward direction than the isotropic medium, while the backward scattering media transport less radiation heat into the forward direction than the isotropic medium.

The effect of the wall reflectivity on the radiative heat transfer is also examined with the medium $F 2$. Figure 7 shows the net radiative heat flux along the centerline of the enclosure

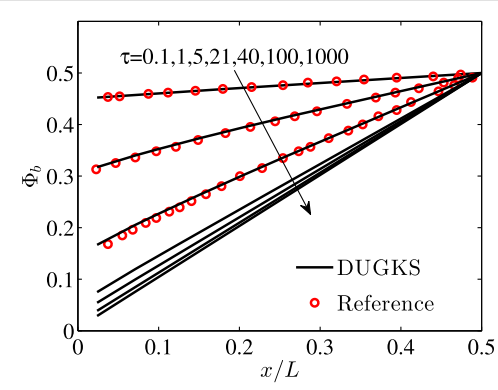

(a)

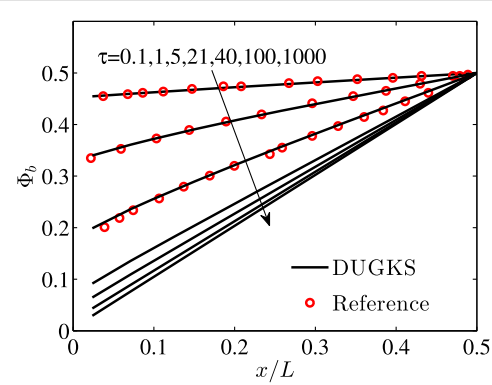

(b)

Fig. 2 Nondimensional temperature distribution for anisotropic media between isothermal plates. Reference data are taken from [43] (a) medium A, (b) medium B 


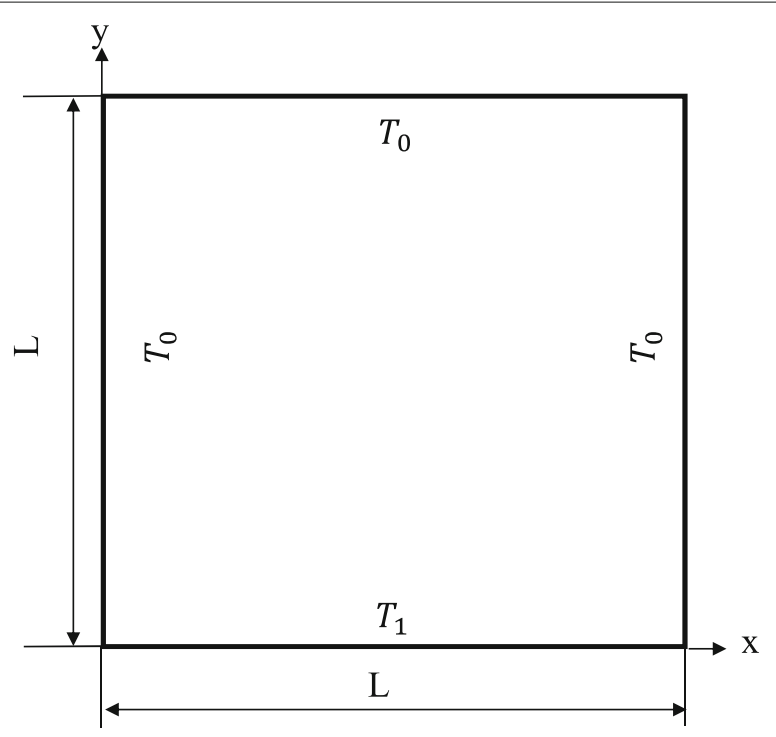

Fig. 3 Schematic of two-dimensional radiation heat transfer with hot wall

at different wall reflectivity. It is noted that with the increasing of $\rho$, the radiative heat flux $q_{y}$ decreases significantly, which is caused by the increasing of the radiative flux that reflects from the walls.

The net radiative heat flux along the centerline $q_{y}$ for different optical thickness with the medium $F 2$ is also shown in Fig. 8. The optical thickness range from $\tau=0.01$ to $\tau=100$ are considered. The DUGKS results agree well with the DOM results [17] from $\tau=0.01$ to $\tau=10$, and have the reasonable results when $\Delta x / \lambda=\tau / N_{y} \geq 1$. Furthermore, in the literature [17], when the optical thickness is larger than 2.5, the DOM scheme should use finer mesh $(52 \times 52)$ to eliminate the error. The DUGKS does not have this problem, as shown in Fig. 8. These results again confirm the capability of the present DUGKS method in simulating $2 \mathrm{D}$ anisotropic radiative scattering problems.

Table 2 Expansion coefficients for the phase function, $C_{j}$

\begin{tabular}{|c|c|c|c|c|c|}
\hline j & isotropy & $F_{1}$ & $F_{2}$ & $B_{1}$ & $B_{2}$ \\
\hline 0 & 1.00000 & 1.00000 & 1.00000 & 1.00000 & 1.00000 \\
\hline 1 & & 2.53602 & 2.00917 & -0.56524 & -1.20000 \\
\hline 2 & & 3.56549 & 1.56339 & 0.29783 & 0.50000 \\
\hline 3 & & 3.97976 & 0.67407 & 0.08571 & \\
\hline 4 & & 4.00292 & 0.22215 & 0.01003 & \\
\hline 5 & & 3.66401 & 0.04725 & 0.00063 & \\
\hline 6 & & 3.01601 & 0.00671 & & \\
\hline 7 & & 2.23304 & 0.00068 & & \\
\hline 8 & & 1.30251 & 0.00005 & & \\
\hline 9 & & 0.53463 & & & \\
\hline 10 & & 0.20136 & & & \\
\hline 11 & & 0.05480 & & & \\
\hline 12 & & 0.01099 & & & \\
\hline$C_{j} / 3$ & 0.00000 & 0.84534 & 0.66972 & -0.18841 & -0.40000 \\
\hline
\end{tabular}




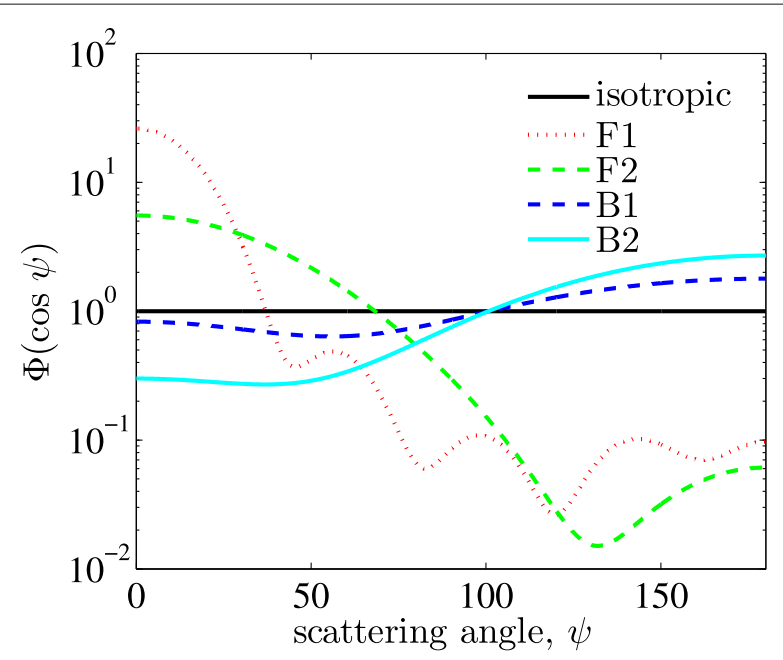

Fig. 4 Scattering phase function

\subsection{Radiation in a square domain with collimated incidence}

To further test the capability of the present DUGKS method in simulating radiative transfer problem with anisotropic scattering media, we now apply it to $2 \mathrm{D}$ square problems with collimated incidence. As shown in Fig. 9, the side length of the square is $L$. All walls and the interior domain are kept cold with the temperature $T_{0}$. A collimate beam $\left(I_{c}=\pi\right)$ is incident through the top boundary. The collimate beam is normal to the top boundary.

The discretization of the physical space is $N_{x} \times N_{y}=26 \times 26$. The solid angle is discretized with $N_{\mu} \times N_{\varphi}=16 \times 16$. The scattering albedo is taken to be $\omega=1.0$. The parameters of phase function for anisotropic scattering media are shown in Table 2 . In this test, with the collimated incidence from the top wall the radiative transfer equation now can be expressed as follows [18],

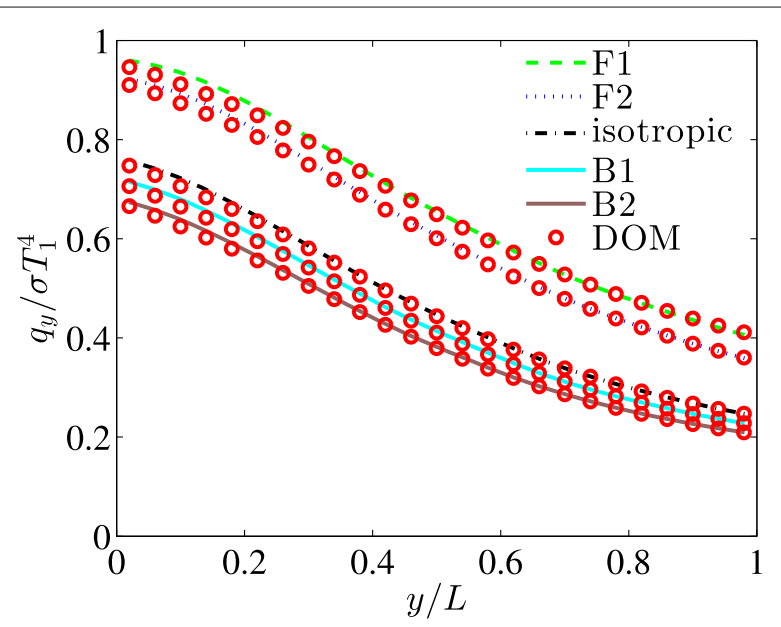

Fig. 5 Effect of anisotropic media on the centerline nondimensional net radiative heat flux in the $y$-direction $(\rho=0, \omega=1.0, \tau=1.0)$ 


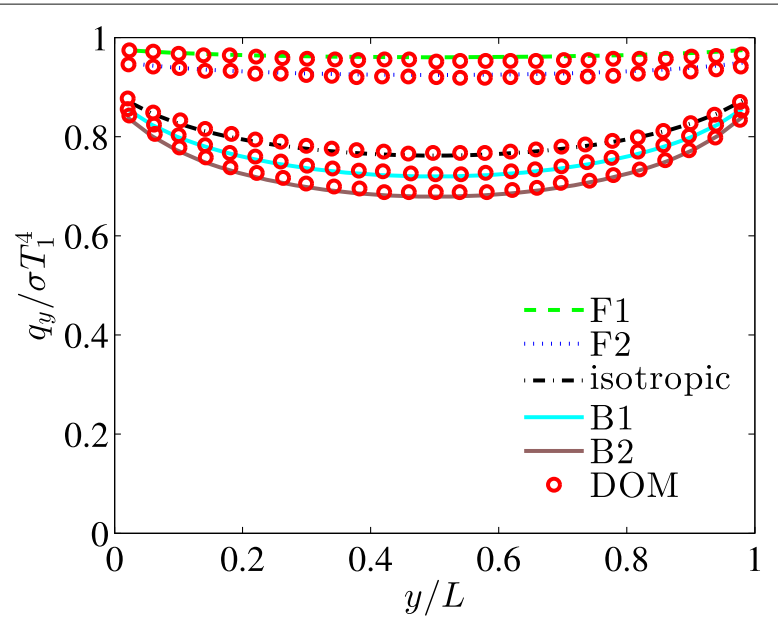

Fig. 6 Nondimensional net radiative heat flux at the hot wall $(\rho=0, \omega=1.0, \tau=1.0)$

$$
\begin{aligned}
\frac{1}{c} \frac{\partial I(\boldsymbol{x}, \boldsymbol{s}, t)}{\partial t}+\boldsymbol{s} \cdot \nabla I(\boldsymbol{x}, \boldsymbol{s}, t) & =-\beta I(\boldsymbol{x}, \boldsymbol{s}, t)+\beta S(\boldsymbol{x}, \boldsymbol{s}, t) \\
& +\frac{\beta \omega}{4 \pi} I_{c} \Phi\left(\boldsymbol{s}_{c}, \boldsymbol{s}\right) \exp \left\{-\left(\tau-\tau_{y}\right)\right\},
\end{aligned}
$$

where $\boldsymbol{s}_{c}$ is incident angle of the beam, and $\tau_{y}=\beta y$.

The walls energy losses for the different phase functions are illustrated in Fig. 10. Fig. 10a shows the reflected components of the radiative flux along the top wall, and the transmitted components of the radiative flux along the bottom wall are shown in Fig. 10b. It is observed that the DUGKS results agree well with the DOM results [18] in all cases. From these figures, the same conclusions as in section 4.2 that backward scattering media reflect more radiative energy while forward scattering media transmit more energy can also be obtained. The side walls energy losses for the different phase functions are shown in Fig. 10c.

The net radiative heat flux along the centerline $q_{y}$ for different anisotropic scattering media and different optical thickness are shown in Fig. 11 and Fig. 12, respectively. All of

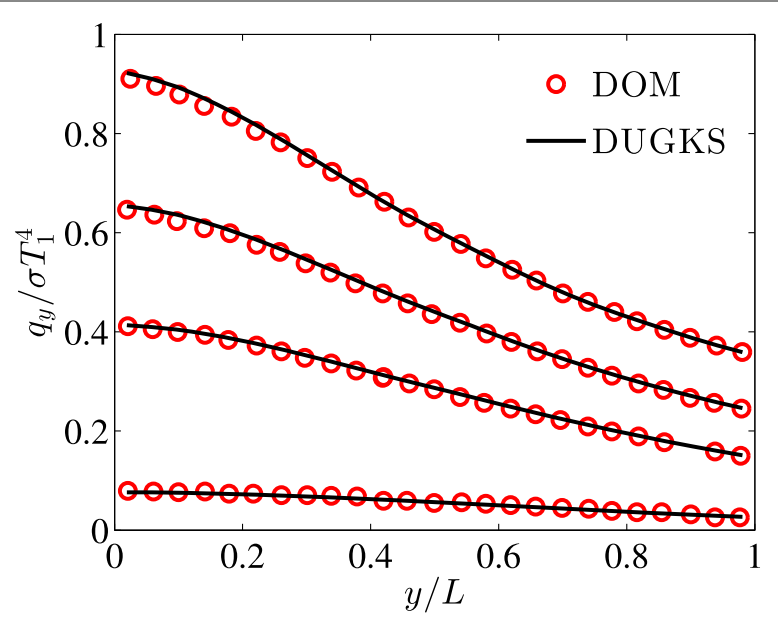

Fig. 7 Effect of wall reflectivity on the centerline nondimensional net radiative heat flux in the $y$-direction (Medium: $F 2, \omega=1.0, \tau=1.0$ ) 


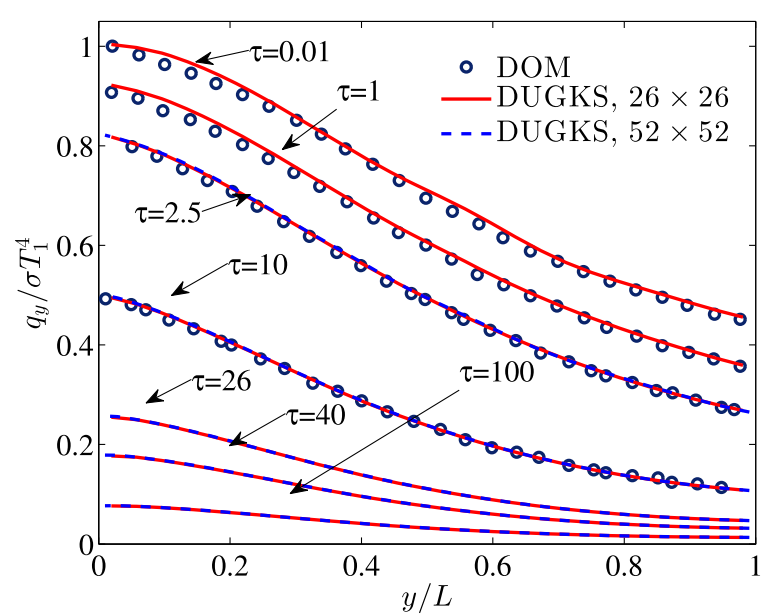

Fig. 8 Effect of optical thickness on the centerline nondimensional net radiative heat flux in the $y$-direction (Medium: $F 2, \rho=0, \omega=1.0$ )

them agree well with the DOM solutions [18]. These tests clearly show that the DUGKS is an accurate solver for radiative transfer with anisotropic scattering media.

\section{Summary}

In present work, we developed a discrete unified gas kinetic scheme for radiative transfer with anisotropic scattering media based on the radiative transfer equation. Due to the complex anisotropic scattering phase function which is calculated by the Legendre polynomial expansion, a simple and efficient iterative approach is employed. The present DUGKS has been validated by a set of radiative transport problems including the radiative transfer in a slab with different wall temperatures, radiative transfer in a square domain with a hot wall, and radiative transfer in a square domain with collimated incidence. All results agree well with other different numerical schemes. In these cases, even if the mesh

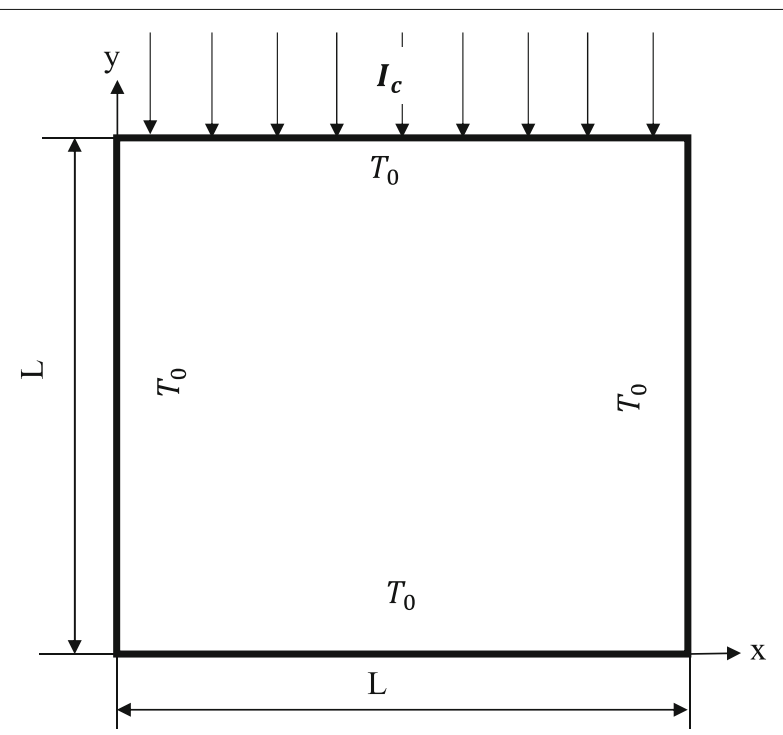

Fig. 9 Schematic of two-dimensional radiation heat transfer with collimated incidence 


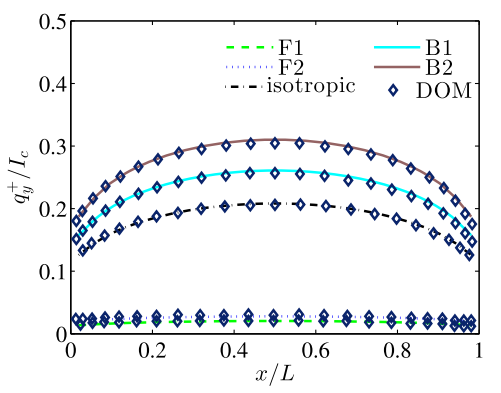

(a)

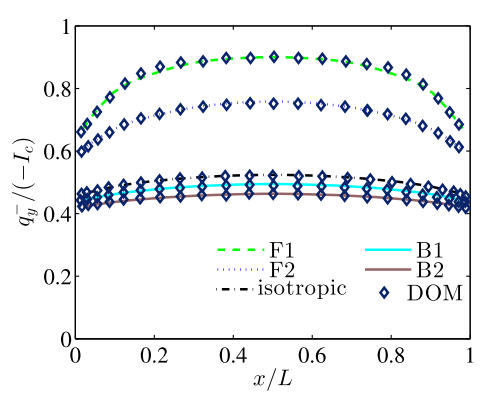

(b)

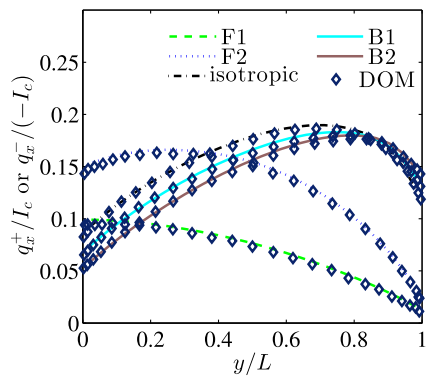

(c)

Fig. 10 Effect of anisotropic media along the walls energy losses $(\rho=0, \omega=1.0, \tau=1.0)$. $\mathbf{a}$ the reflected flux loss on top wall, $\mathbf{b}$ the transmitted flux loss on bottom wall, $\mathbf{c}$ the flux loss on side walls

size is larger than the photon mean free path, the results predicted by the present scheme are still reliable. As the scheme has the nice unified preserving properties and the mesh size is not restricted by the photon mean free path, the present DUGKS will be an efficient and accurate tool to describe the multiscale anisotropic radiative heat transfer. It will also be easy to handle more complex radiative transfer problems with unstructured meshes, due to its finite volume property.

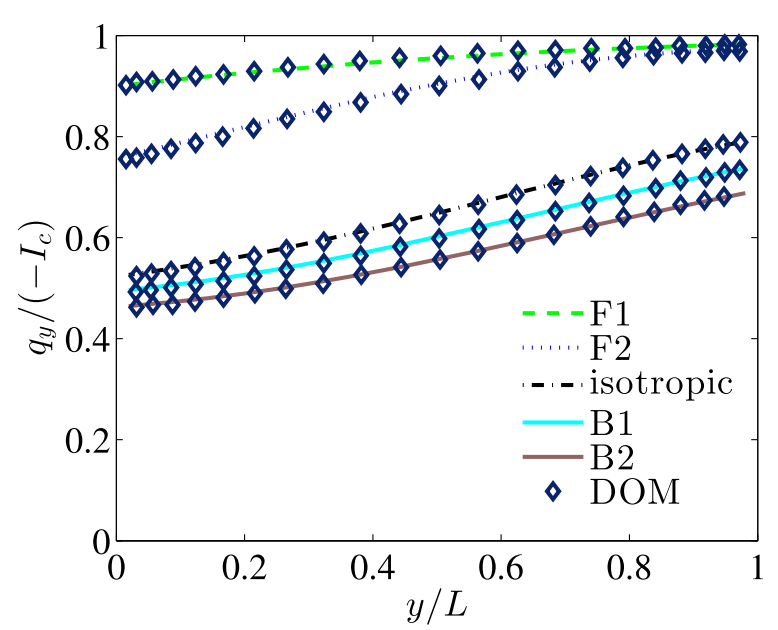

Fig. 11 Effect of anisotropic media on the centerline nondimensional net radiative heat flux in the $y$-direction ( $\rho=0, \omega=1.0, \tau=1.0$ ) 


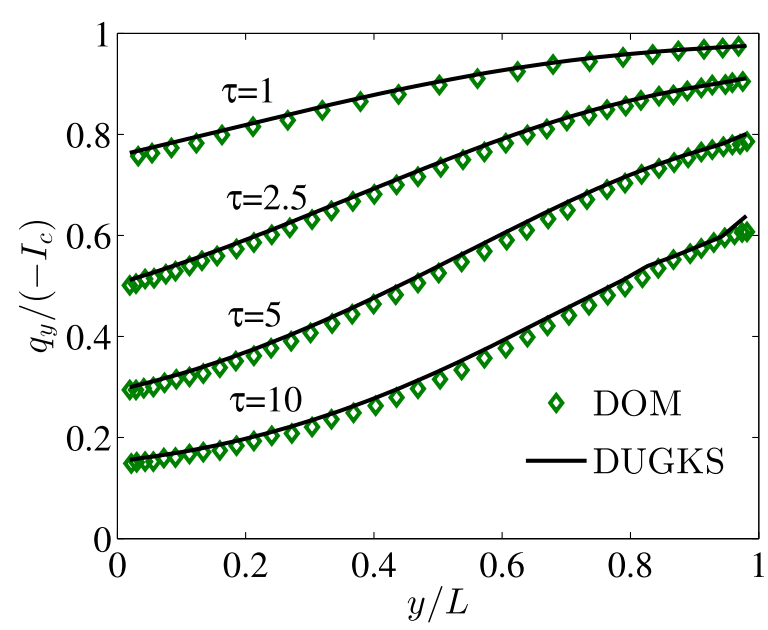

Fig. 12 Effect of optical thickness on the centerline nondimensional net radiative heat flux in the $y$-direction (Medium: $F 2, \rho=0, \omega=1.0$ )

Availability of supporting data

Not applicable.

\section{Acknowledgments}

This work was supported by the National Key R\&D Program of China (No. 2018YFE0180900) and the Fundamental Research Funds for the Central Universities (No. 2019kfyXMBZ040).

\section{Authors' contributions}

The research output is coming from our joint effort. All authors read and approved the final manuscript.

\section{Funding}

The National Key R\&D Program of China (No. 2018YFE0180900) and the Fundamental Research Funds for the Central Universities (No. 2019kfyXMBZ040).

\section{Availability of data and materials}

All data generated or analyzed during this study are included in this published article.

\section{Competing interests}

The authors declare that they have no competing interests.

\section{Author details}

${ }^{1}$ State Key Laboratory of Coal Combustion, School of Energy and Power Engineering, Huazhong University of Science and Technology, 430074 Wuhan, China. ${ }^{2}$ Department of Nuclear Engineering and Technology, School of Energy and Power Engineering, Huazhong University of Science and Technology, 430074 Wuhan, China.

Received: 22 October 2019 Accepted: 19 December 2019

Published online: 10 January 2020

\section{References}

1. Luo XP, Wang CH, Zhang Y, Yi HL, Tan HP (2018) Multiscale solutions of radiative heat transfer by the discrete unified gas kinetic scheme. Phys Rev E 97(6):063302

2. Guo Z, Kumar S (2001) Discrete-ordinates solution of short-pulsed laser transport in two-dimensional turbid media. Appl Opt 40(19):3156-3163

3. Baek SW, Kim MY (1997) Analysis of radiative heating of a rocket plume base with the finite-volume method. Int J Heat Mass Transfer 40(7):1501-1508

4. Naraghi M, Dunn S, Coats D (2005) Modeling of radiation heat transfer in liquid rocket engines. In: 41st AIAA/ASME/SAE/ASEE Joint Propulsion Conference \& Exhibit. p 3935

5. Broc A, Joly V, Lafon JP, Marmignon C (1998) Nonequilibrium radiative hypersonic flows: aerospace applications. Astrophys Space Sci 260(1-2):29-43

6. Liou KN (2002) An Introduction to Atmospheric Radiation. vol. 84. Elsevier, Orlando

7. Chahlafi M, Bellet F, Fichot F, Taine J (2012) Radiative transfer within non beerian porous media with semitransparent and opaque phases in non equilibrium: Application to reflooding of a nuclear reactor. Int J Heat Mass Transfer 55(13-14):3666-3676

8. Badinand T, Fransson TH (2003) Radiative heat transfer in film-cooled liquid rocket engine nozzles. J Thermophys Heat Transf 17(1):29-34 
9. Liu J, Shang H, Chen Y, Wang T (1996) Investigation of rocket plume radiation by discrete ordinates method. In: 34 th Aerospace Sciences Meeting and Exhibit. American Institute of Aeronautics and Astronautics. https://doi.org/10. 2514/6.1996-348

10. Hunter B, Guo Z (2016) Improved treatment of anisotropic scattering in radiation transfer analysis using the finite volume method. Heat Trans Eng 37(3-4):341-350

11. Williams M (1984) The effect of anisotropic scattering on the radiant heat flux through an aerosol. J Phys D Appl Phys 17(8):1617

12. Marakis J, Papapavlou C, Kakaras E (2000) A parametric study of radiative heat transfer in pulverised coal furnaces. Int J Heat Mass Transfer 43(16):2961-2971

13. DER J, NELSON D (1985) Internal radiative heating from aluminum oxide particles in solid propellant rocket motors. In: 21st Joint Propulsion Conference. American Institute of Aeronautics and Astronautics. p 1397. https://doi.org/10. 2514/6.1985-1397

14. Subramaniam S, Mengüç MP (1991) Solution of the inverse radiation problem for inhomogeneous and anisotropically scattering media using a monte carlo technique. Int J Heat Mass Transfer 34(1):253-266

15. Farmer JT, Howell JR (1994) Monte carlo prediction of radiative heat transfer in inhomogeneous, anisotropic, nongray media. J Thermophys Heat Tran 8(1):133-139

16. Liming $\mathrm{R}$, Jinmo $\mathrm{H}$, Heping $\mathrm{T}$ (2002) Monte carlo method to radiative transfer in two-dimensional scattering rectangular enclosures. J Combust Sci Technol 8(5):390-394

17. Kim TK, Lee H (1988) Effect of anisotropic scattering on radiative heat transfer in two-dimensional rectangular enclosures. Int J Heat Mass Transfer 31(8):1711-1721

18. Kim TK, Lee HS (1989) Radiative transfer in two-dimensional anisotropic scattering media with collimated incidence. J Quant Spectrosc Radiative Tran 42(3):225-238

19. Hunter B, Guo Z (2013) Comparison of quadrature schemes in dom for anisotropic scattering radiative transfer analysis. Numer Heat Tran Part B Fundam 63(6):485-507

20. Jinbo H, Liming R, Heping $T$ (2003) Effect of anisotropic scattering on radiative heat transfer in two-dimensional rectangular media. J Quant Spectrosc Radiative Tran 78(2):151-161

21. Chai JC, Parthasarathy G, Lee HS, Patankar SV (1995) Finite volume radiative heat transfer procedure for irregular geometries. J Thermophys Heat Tran 9(3):410-415

22. Kim SH, Huh KY (2000) A new angular discretization scheme of the finite volume method for 3-d radiative heat transfer in absorbing, emitting and anisotropically scattering media. Int J Heat Mass Transfer 43(7):1233-1242

23. Larsen EW, Morel JE, Miller Jr WF (1987) Asymptotic solutions of numerical transport problems in optically thick, diffusive regimes. J Comput Phys 69(2):283-324

24. Larsen EW, Morel J. E. (1989) Asymptotic solutions of numerical transport problems in optically thick, diffusive regimes ii. J Comput Phys 83(1):212-236

25. Jin S, Levermore D (1991) The discrete-ordinate method in diffusive regimes. Transport Theory \& Statistical Physics 20(5-6):413-439

26. Jin S, Levermore CD (1993) Fully-discrete numerical transfer in diffusive regimes. Tran Theory Stat Phys 22(6):739-791

27. Klar A (1998) An asymptotic-induced scheme for nonstationary transport equations in the diffusive limit. SIAM J Numer Anal 35(3)

28. Shi J, Pareschi L, Toscani G (2000) Uniformly accurate diffusive relaxation schemes for multiscale transport equations. SIAM J Numer Anal 38(3):913-936

29. Mieussens $L$ (2013) On the asymptotic preserving property of the unified gas kinetic scheme for the diffusion limit of linear kinetic models. J Comput Phys 253:138-156

30. Li W, Liu C, Zhu Y, Zhang J, Xu K (2019) Unified gas-kinetic wave-particle methods iii: Multiscale photon transport. arXiv preprint arXiv:1904.01787. https://arxiv.xilesou.top/abs/1904.01787

31. Sun W, Jiang S, Xu K (2015) An asymptotic preserving unified gas kinetic scheme for gray radiative transfer equations. J Comput Phys 285:265-279

32. Guo Z, Xu K, Wang R (2013) Discrete unified gas kinetic scheme for all knudsen number flows: Low-speed isothermal case. Phys Rev E 88(3):033305

33. Guo Z, Wang R, Xu K (2015) Discrete unified gas kinetic scheme for all knudsen number flows. ii. thermal compressible case. Phys Rev E 91(3):033313

34. Guo Z, Li J, Xu K (2019) On unified preserving properties of kinetic schemes. arXiv preprint arXiv:1909.04923. https:// arxiv.xilesou.top/abs/1909.04923

35. Chu CM, Churchill SW (1955) Representation of the angular distribution of radiation scattered by a spherical particle. JOSA 45(11):958-962

36. Clark GC, Chu CM, Churchill SW (1957) Angular distribution coefficients for radiation scattered by a spherical particle. JOSA 47(1):81-84

37. Modest MF (2013) Radiative Heat Transfer. Academic press, Merced

38. Tan ZM, Hsu PF (2001) An integral formulation of transient radiative transfer. J Heat Transf 123(3):466-475

39. Guo Z, Xu K (2016) Discrete unified gas kinetic scheme for multiscale heat transfer based on the phonon boltzmann transport equation. Int J Heat Mass Transfer 102:944-958

40. Van Leer B (1977) Towards the ultimate conservative difference scheme. iv. a new approach to numerical convection. J Comput Phys 23(3):276-299

41. Xu K, Huang JC (2010) A unified gas-kinetic scheme for continuum and rarefied flows. J Comput Phys 229(20):7747-7764

42. Abramowitz M, Stegun IA (1965) Handbook of mathematical functions. Dover publications, New York

43. Modest M, Azad F (1980) The influence and treatment of mie-anisotropic scattering in radiative heat transfer. J Heat Transf 102(1):92-98

\section{Publisher's Note}

Springer Nature remains neutral with regard to jurisdictional claims in published maps and institutional affiliations. 